Article

\title{
Neutrosophic Linear Equations and Application in Traffic Flow Problems
}

\author{
Jun Ye
}

Department of Electrical and Information Engineering, Shaoxing University, 508 Huancheng West Road, Shaoxing 312000, China; yehjun@aliyun.com or yejun@usx.edu.cn; Tel.: +86-575-8832-7323

Received: 2 October 2017; Accepted: 30 November 2017; Published: 1 December 2017

\begin{abstract}
A neutrosophic number (NN) presented by Smarandache can express determinate and/or indeterminate information in real life. $\mathrm{NN}(z=a+u I)$ consists of the determinate part $a$ and the indeterminate part $u I$ for $a, u \in R$ ( $R$ is all real numbers) and indeterminacy $I$, and is very suitable for representing and handling problems with both determinate and indeterminate information. Based on the concept of NNs, this paper presents for first time the concepts of neutrosophic linear equations and the neutrosophic matrix, and introduces the neutrosophic matrix operations. Then, we propose some solving methods, including the substitution method, the addition method, and the inverse matrix method, for the system of neutrosophic linear equations or the neutrosophic matrix equation. Finally, an applied example about a traffic flow problem is provided to illustrate the application and effectiveness of handling the indeterminate traffic flow problem by using the system of neutrosophic linear equations.
\end{abstract}

Keywords: neutrosophic number; neutrosophic linear equation; neutrosophic matrix; traffic flow

\section{Introduction}

The condensed traffic flow along with the increasing number of vehicles is increasingly complex and uncertain in actual road traffic situations [1]. Since fuzzy logic and fuzzy algebra were introduced by Zadeh [2] in 1965, fuzzy theory has been successfully applied in a wide range of subject fields [1,3,4]. The main reason for this widespread application is that it can process various vague or uncertain data and provide results that are suitable for decision making. In a fuzzy set, fuzzy numbers are proven to be very suitable for expressing vague, imprecise, uncertain values [3,4]. Then, fuzzy numbers can be defined by a membership/truth function, which specifies the membership/truth degree for each element $x$ from the universe $X$. In many real situations (e.g., traffic flow), the information obtained by decision makers may be partial determinacy and/or partial indeterminacy due to a lack of data, time pressure, measurement errors, or the decision makers' limited attention and knowledge. However, the fuzzy sets/numbers cannot represent data with both determinate and indeterminate information. To express it, Smarandache [5-7] first proposed the concept of a neutrosophic number (NN) as a subclass of neutrosophy. NN is composed of the determinate part $a$ and the indeterminate part $u I$, denoted by $z=a+u I$ for $a, u \in R$ ( $R$ is all real numbers), where $I$ is indeterminacy. Hence, NN is much more suitable for representing and handling real problems with both determinate and indeterminate information. Recently, NNs have been applied to decision making [8,9] and fault diagnoses [10,11].

Then, Smarandache [12] further proposed the concept of the neutrosophic function, neutrosophic precalculus, and neutrosophic calculus in 2015 as another subclass of neutrosophy to deal with various indeterminate problems in the real world. In general, a neutrosophic function (thick function) $f: R$ $\rightarrow F(R)$ can be defined as the forms of a closed interval function $f(x)=\left[f_{1}(x), f_{2}(x)\right]$, an open interval function $f(x)=\left(f_{1}(x), f_{2}(x)\right)$, and semi-open/semi-closed interval functions $f(x)=\left[f_{1}(x), f_{2}(x)\right) / f(x)$ $=\left(f_{1}(x), f_{2}(x)\right]$. Then, Ye et al. [13] applied the neutrosophic function to the expression of the joint 
roughness coefficient (JRC) and the shear strength in rock mechanics. However, the neutrosophic functions introduced in [12,13] are interval functions (thick function), but they cannot express and handle actual problems containing NN information. Furthermore, NNs were also applied to expressions and analyses of rock joint roughness coefficient in rock mechanics [14-16]. Further, Ye [17] put forth an NN linear programming method to obtain optimal solutions of NN linear programming problems under $\mathrm{NN}$ environments. Unfortunately, there is no study on neutrosophic linear equations and their solution methods in the currently existing literature. It is obvious that existing fuzzy sets/numbers cannot express neutrosophic linear equations with both determinate and indeterminate information (NNs).

In this paper, based on NNs we propose for the first time the new concepts of neutrosophic linear equations and the neutrosophic matrix, and the solving methods of the system of neutrosophic linear equations by using the substitution method, the addition method, and the inverse matrix method. To do so, the rest of this paper is organized as follows. Section 2 describes some basic concepts and operational laws of NNs. In Section 3, we propose some new concepts in neutrosophic linear equations and neutrosophic matrices and introduce some neutrosophic matrix operations. Section 4 introduces the solving methods of the system of neutrosophic linear equations or the neutrosophic matrix equation by using the substitution, addition, and inverse matrix methods. In Section 5, an applied example about a traffic flow problem is provided to illustrate the application and effectiveness of handling the indeterminate traffic flow problem by means of a system of neutrosophic linear equations. Section 6 contains some conclusions and further research.

\section{Neutrosophic Numbers and Their Operational Laws}

Smarandache [5-7] presented an NN concept which is composed of the determinate part $a$ and the indeterminate part $u I$, and is denoted by $z=a+u I$ for $a, u \in R$ ( $R$ is all real numbers), where $I$ is indeterminacy.

For example, if an $\mathrm{NN}$ is $z=3+2 I$ for $I \in[0,0.2]$, it is equivalent to $z \in[3,3.4]$, which means that its determinate part is 3 and its indeterminate part is $2 I=[0,0.4]$ for the indeterminacy $I \in[0,0.2]$ and the possibility for the number " $z$ " is within the interval [3,3.4], and then for $I \in[0.1,0.3]$, the possible value of $z$ is within the interval [3.2,3.6]. As another example, the possible value of the fraction $5 / 3$ is within the interval $[1.66,1.67]$, which can be expressed as the neutrosophic number $z=1.66+0.01 \mathrm{I}$ for $I \in[0,1]$.

It is obvious that an $\mathrm{NN} z=a+u I$ is equivalent to a changeable interval number $z=[a+u \cdot$ infI, $a+u \cdot \sup I]$ for $a, u \in R$ and $I \in$ [infI, sup $I]$ (possible changeable range of indeterminacy). In special cases, $z$ can be expressed as the indeterminate part $z=u I$ if $a=0$ for the worst scenario; while $z$ can also be expressed as the determinate part $z=a$ if $u I=0$ for the best scenario.

For two NNs $z_{1}=a_{1}+u_{1} I$ and $z_{2}=a_{2}+u_{2} I$ for $a_{1}, u_{1}, a_{2}, u_{2} \in R$, their basic operational laws for $I \in[$ infI, sup $I]$ are defined as follows [17]:

(1) $z_{1}+z_{2}=a_{1}+a_{2}+\left(u_{1}+u_{2}\right) I=\left[a_{1}+a_{2}+u_{1} \inf I+u_{2} \operatorname{infI}, a_{1}+a_{2}+u_{1} \sup I+u_{2} \sup I\right]$

(2) $z_{1}-z_{2}=a_{1}-a_{2}+\left(u_{1}-u_{2}\right) I=\left[a_{1}-a_{2}+u_{1} \inf I-u_{2} \inf I, a_{1}-a_{2}+u_{1} \sup I-u_{2} \sup I\right]$;

$z_{1} \times z_{2}=a_{1} a_{2}+\left(a_{1} u_{2}+a_{2} u_{1}\right) I+u_{1} u_{2} I^{2}$$$
=\left[\begin{array}{c}
\min \left(\begin{array}{c}
\left(a_{1}+u_{1} \inf I\right)\left(a_{2}+u_{2} \inf I\right),\left(a_{1}+u_{1} \inf I\right)\left(a_{2}+u_{2} \sup I\right), \\
\left(a_{1}+u_{1} \sup I\right)\left(a_{2}+u_{2} \inf I\right),\left(a_{1}+u_{1} \sup I\right)\left(a_{2}+u_{2} \sup I\right)
\end{array}\right), \\
\max \left(\begin{array}{c}
\left(a_{1}+u_{1} \inf I\right)\left(a_{2}+u_{2} \inf I\right),\left(a_{1}+u_{1} \inf I\right)\left(a_{2}+u_{2} \sup I\right), \\
\left(a_{1}+u_{1} \sup I\right)\left(a_{2}+u_{2} \inf I\right),\left(a_{1}+u_{1} \sup I\right)\left(a_{2}+u_{2} \sup I\right)
\end{array}\right)
\end{array}\right] ;
$$

(4)

$$
=\left[\begin{array}{c}
\frac{z_{1}}{z_{2}}=\frac{a_{1}+u_{1} I}{a_{2} I}=\frac{\left[a_{1}+u_{1} \inf I, a_{1}+u_{1} \sup I\right]}{\left.a_{2}+u_{2} \inf I, a_{2}+u_{2} \sup I\right]} \\
\min \left(\frac{a_{1}+u_{1} \inf I}{a_{2}+u_{2} \sup I}, \frac{a_{1}+u_{1} \operatorname{infI} I}{a_{2}+u_{2} \operatorname{infI}}, \frac{a_{1}+u_{1} \sup I}{a_{2}+u_{2} \sup I}, \frac{a_{1}+u_{1} \sup I}{a_{2}+u_{2} \operatorname{infI}}\right), \\
\max \left(\frac{a_{1}+u_{1} \inf I}{a_{2}+u_{2} \sup I}, \frac{a_{1}+u_{1} \inf I}{a_{2}+u_{2} \operatorname{infI} I}, \frac{a_{1}+u_{1} \sup I}{a_{2}+u_{2} \sup I}, \frac{a_{1}+u_{1} \sup I}{a_{2}+u_{2} \operatorname{infI} I}\right)
\end{array}\right]
$$




\section{Neutrosophic Linear Equations and Neutrosophic Matrices}

\subsection{Neutrosophic Linear Equations}

Definition 1. A neutrosophic linear equation in $n$ variables (unknowns) $x_{1}, x_{2}, \ldots, x_{n}$ is defined as the following form:

$$
z_{1} x_{1}+z_{2} x_{2}+\ldots+z_{n} x_{n}=z
$$

where $z, z_{j} \in Z(j=1,2, \ldots, n)$ are the given real $N N$ s and $Z$ are all NNs.

For example, if the neutrosophic linear equation is $2 I x_{1}+3 x_{2}=6$ for $I \in[1,2]$, then it indicates the indeterminate area between two lines $2 x_{1}+3 x_{2}=6$ and $4 x_{1}+3 x_{2}=6$, which can be represented as one neutrosophic function $[2,4] x_{1}+3 x_{2}=6$, and then if the indeterminacy $I \in[2,4]$ is considered as a possible interval range, then the neutrosophic linear equation can be also described as the indeterminate area between two lines $4 x_{1}+3 x_{2}=6$ and $8 x_{1}+3 x_{2}=6$ and be represented as another neutrosophic function $[4,8] x_{1}+3 x_{2}=6$.

Definition 2. A system of $m$ neutrosophic linear equations in $n$ variables (unknowns) $x_{1}, x_{2}, \ldots, x_{n}$ is defined as a family of neutrosophic linear equations:

$$
\begin{gathered}
z_{11} x_{1}+z_{12} x_{2}+\ldots+z_{1 n} x_{n}=z_{1} \\
z_{21} x_{1}+z_{22} x_{2}+\ldots+z_{2 n} x_{n}=z_{2} \\
\vdots \\
z_{m 1} x_{1}+z_{m 2} x_{2}+\ldots+z_{m n} x_{n}=z_{m}
\end{gathered}
$$

For convenient expression, the above system can be written concisely as

$$
\sum_{j=1}^{n} z_{i j} x_{j}=z_{i} \text { for } i=1,2, \ldots, m
$$

Definition 3. Let

$$
\left[\begin{array}{cccc}
z_{11} & z_{12} & \cdots & z_{1 n} \\
z_{21} & z_{22} & \cdots & z_{2 n} \\
\vdots & & & \vdots \\
z_{m 1} & z_{m 2} & \cdots & z_{m n}
\end{array}\right]
$$

which is called the neutrosophic coefficient matrix of the system, while

$$
\left[\begin{array}{ccccc}
z_{11} & z_{12} & \cdots & z_{1 n} & z_{1} \\
z_{21} & z_{22} & \cdots & z_{2 n} & z_{2} \\
\vdots & & & & \vdots \\
z_{m n} & z_{m n} & \cdots & z_{m n} & z_{m}
\end{array}\right]
$$

is called the neutrosophic augmented matrix of the system. 
Let us write

$$
A=\left[\begin{array}{cccc}
z_{11} & z_{12} & \cdots & z_{1 n} \\
z_{21} & z_{22} & \cdots & z_{2 n} \\
\vdots & & & \vdots \\
z_{m 1} & z_{m 2} & \cdots & z_{m n}
\end{array}\right], X=\left[\begin{array}{c}
x_{1} \\
x_{2} \\
\vdots \\
x_{n}
\end{array}\right] \text {, and } B=\left[\begin{array}{c}
z_{1} \\
z_{2} \\
\vdots \\
z_{m}
\end{array}\right]
$$

Then, the system of neutrosophic linear equations for Equation (2) can be expressed by the following form of the neutrosophic matrix equation:

$$
A X=B .
$$

\subsection{Operations of Neutrosophic Matrices}

For neutrosophic matrices, all the classical matrix operations (e.g., matrix addition, matrix subtraction, and scalar multiplication, matrix transpose, matrix inverse, and so on) can be used to perform the neutrosophic matrix operations.

Example 1. Assume there are two neutrosophic matrices $A$ and B:

$$
A=\left[\begin{array}{cc}
I & 3 \\
5 I & 9
\end{array}\right] \text { and } B=\left[\begin{array}{cc}
I & 2 I \\
3 I & 5
\end{array}\right]
$$

Thus, we can introduce the following matrix operations:

(1) $A+B: A+B=\left[\begin{array}{cc}2 I & 3+2 I \\ 8 I & 14\end{array}\right]$;

(2) $A-B: A-B=\left[\begin{array}{cc}0 & 3-2 I \\ 2 I & 4\end{array}\right]$;

(3) $3 A: 3 A=\left[\begin{array}{cc}3 I & 9 \\ 15 I & 27\end{array}\right]$;

(4) $A^{T}: A^{T}=\left[\begin{array}{cc}I & 5 I \\ 3 & 9\end{array}\right]$;

(5) $A \times B: A \times B=\left[\begin{array}{cc}(9+I) I & 15+2 I^{2} \\ (27+5 I) I & 45+10 I^{2}\end{array}\right]$;

(6) $A^{-1}: A^{-1}=\left[\begin{array}{cc}-3 /(2 I) & 1 /(2 I) \\ 5 / 6 & -1 / 6\end{array}\right]$.

\section{Solving a System of Neutrosophic Linear Equations}

To determine if such a system of neutrosophic linear equations has a solution, one needs to find out if there exists a group of numbers for $x_{1}, x_{2}, \ldots, x_{n}$ which can satisfy each of the equations in Equation (2) simultaneously. The system is consistent if it has a solution. Otherwise, the system is inconsistent.

Generally, a system of neutrosophic linear equations can be solved by solving methods of systems of classical linear equations, such as the substitution method, the addition method, and the inversion matrix method. In this section, two examples of the system of neutrosophic linear equations show solving processes by using the substitution method, the addition method, and the inverse of a square matrix. 
Example 2. Let us consider the following system of neutrosophic linear equations in two variables:

$$
\begin{gathered}
3 I x_{1}+2 x_{2}=2 \text { (the first equation), } \\
5 I x_{1}+7 x_{2}=3 \text { (the second equation). }
\end{gathered}
$$

Then, we use the substitution method, the addition method, and the inverse matrix method, respectively, to solve the system of neutrosophic linear equations.

(1) Using the substitution method

Solve the first equation for $x_{1}$ :

$x_{1}=\left(2-2 I x_{2}\right) /(3 I)$.

Substitute $x_{1}=\left(2-2 I x_{2}\right) /(3 I)$ into the second equation:

$5 I\left(2-2 I x_{2}\right) /(3 I)+7 x_{2}=3$.

Then, $5\left(2-2 I x_{2}\right) / 3+7 x_{2}=3$.

Thus, the solution for $x_{2}$ is $x_{2}=-1 / 11$, and then we obtain $3 I x_{1}-2 / 11=2$ from the first equation.

Hence, the solution of the system is $x_{1}=8 /(11 I)$ and $x_{2}=-1 / 11$.

(2) Using the addition method

Multiply the first equation by -5 and the second equation by 3 :

$-15 I x_{1}-10 x_{2}=-10$,

$15 I x_{1}+21 x_{2}=9$.

Add the two equations:

$11 x_{2}=-1$.

Thus, $x_{2}=-1 / 11$, and then $3 I x_{1}-2 / 11=2$ for the first equation.

Therefore, the solution of the system is $x_{1}=8 /(11 I)$ and $x_{2}=-1 / 11$.

(3) Using the inverse matrix method

If $A X=B$ is a neutrosophic matrix equation composed of $m$ neutrosophic linear equations in $n$ unknowns and if the inverse $A^{-1}$ exists (i.e., $A$ is nonsingular), by multiplying both sides of the equation $A X=B$ by $A^{-1}$ (on the left), we obtain the desired solution to the neutrosophic matrix equation as $X=A^{-1} B$.

Let us write the system in Example 2 by the neutrosophic matrices:

$$
A=\left[\begin{array}{ll}
3 I & 2 \\
5 I & 7
\end{array}\right], X=\left[\begin{array}{l}
x_{1} \\
x_{2}
\end{array}\right], \text { and } B=\left[\begin{array}{l}
2 \\
3
\end{array}\right]
$$

Then, the system of neutrosophic linear equations can be written in the form of the neutrosophic matrix equation:

$$
A X=B .
$$

Since the determinant of the neutrosophic matrix $A$ can be evaluated by $|A|=21 I-10 I=11 I$, the inverse of the neutrosophic matrix $A$ is as follows:

$$
A^{-1}=\left[\begin{array}{cc}
7 /(11 I) & -2 /(11 I) \\
-5 / 11 & 3 / 11
\end{array}\right] \text {. }
$$


Using this result, by multiplying both sides of Equation (4) by $A^{-1}$ (on the left), we find that the solution of the system is

$$
X=A^{-1} B=\left[\begin{array}{cc}
7 /(11 I) & -2 /(11 I) \\
-5 / 11 & 3 / 11
\end{array}\right]\left[\begin{array}{l}
2 \\
3
\end{array}\right]=\left[\begin{array}{c}
8 /(11 I) \\
-1 /(11)
\end{array}\right] .
$$

Hence, the solution of the system is $x_{1}=8 /(11 I)$ and $x_{2}=-1 / 11$.

Especially, if $I \in[2,3]$ is considered as a possible interval of the indeterminacy $I$, then the solution of the system is $x_{1}=[0.2424,0.3636]$ and $x_{2}=-0.0909$. If $I=2$, the system is degenerated to the system of classical linear equations, and its solution is given by $x_{1}=0.3636$ and $x_{2}=-0.0909$.

It is obvious that the system of neutrosophic linear equations may be written in a compact form with the help of matrices. Hence, one will use this matrix equation representation and apply the inverse matrix method for solving this neutrosophic matrix equation easily by the MATLAB software.

To illustrate the solving process of the neutrosophic matrix equation by the MATLAB software, we use the inverse matrix method to solve the neutrosophic matrix equation by the following example.

Example 3. Assume that there is the following neutrosophic matrix equation:

$$
\left[\begin{array}{ccc}
2 & -4 & 1 \\
-3 & 6 & -5 \\
4 & -3 & 7
\end{array}\right]\left[\begin{array}{l}
x_{1} \\
x_{2} \\
x_{3}
\end{array}\right]=\left[\begin{array}{c}
4 I \\
-2 I \\
3 I
\end{array}\right]
$$

where $A=\left[\begin{array}{ccc}2 & -4 & 1 \\ -3 & 6 & -5 \\ 4 & -3 & 7\end{array}\right], X=\left[\begin{array}{l}x_{1} \\ x_{2} \\ x_{3}\end{array}\right]$, and $B=\left[\begin{array}{c}4 I \\ -2 I \\ 3 I\end{array}\right]$. Then, we need to find the solution of $X=\left[x_{1}, x_{2}, x_{3}\right]^{T}$.

Thus, we apply the inverse matrix method for solving this neutrosophic matrix equation by the MATLAB software, which is shown in the following program:

$\%$ The MATLAB solution program of the neutrosophic matrix equation

clc

syms I;

$A=[2,-4,1 ;-3,6,-5 ; 4,-3,7]$;

$B=\left[4^{*} I,-2 * I, 3^{*} I\right]$;

$C=\operatorname{inv}(A)$

$X=C \times B^{\prime}$

Running results of the program are as follows:

$$
\begin{aligned}
& \mathrm{C}= \\
& \begin{array}{lll}
0.7714 & 0.7143 & 0.4000
\end{array} \\
& \begin{array}{lll}
0.0286 & 0.2857 & 0.2000
\end{array} \\
& \begin{array}{lll}
-0.4286 & -0.2857 \quad 0
\end{array} \\
& X= \\
& \left(20^{*} I\right) / 7 \\
& \text { I/7 } \\
& -\left(8^{*} I\right) / 7
\end{aligned}
$$

If $I \in[1,2]$ is considered as a possible interval of the indeterminacy $I$, then the solution of the system is $x_{1}=[2.8571,5.7143], x_{2}=[0.1429,0.2857]$, and $x_{3}=[-1.1429,-2.2857]$. Especially, when $I=1$, 
the system is degenerated to the system of classical linear equations and its solution is given by $x_{1}=2.8571, x_{2}=0.1429$, and $x_{3}=-1.1429$.

Obviously, the classical linear equations and solution are special cases of the neutrosophic linear equations and solution. However, the classical linear equations cannot deal with real problems with indeterminate information, while the neutrosophic linear equations can do them with determinate and/or indeterminate information.

\section{Application of a Traffic Flow Problem}

In some city, the downtown traffic flows of four roads are shown in Figure 1 during the rush hours on a typical weekday. In Figure 1, the arrows indicate the direction of traffic flow on each one-way road, and the average number of vehicles per hour entering and leaving each intersection is shown beside each road. Road 3 and Road 4 can handle up to 2000 vehicles per hour without causing congestion, while the maximum capacity of both Road 1 and Road 2 is 1000 vehicles per hour. The flow of traffic is controlled by the traffic lights, which are installed at each of the four intersections. Then, there exists some indeterminate traffic flow on each one-way road in Figure 1. If the neutrosophic number $\mathrm{z}=400+I$ is expressed as the determinate and indeterminate information of Road 1 , where the indeterminacy $I$ will ensure no traffic congestion, then we need to find the ranges of traffic flows for the unknowns $x_{1}, x_{2}, x_{3}$.

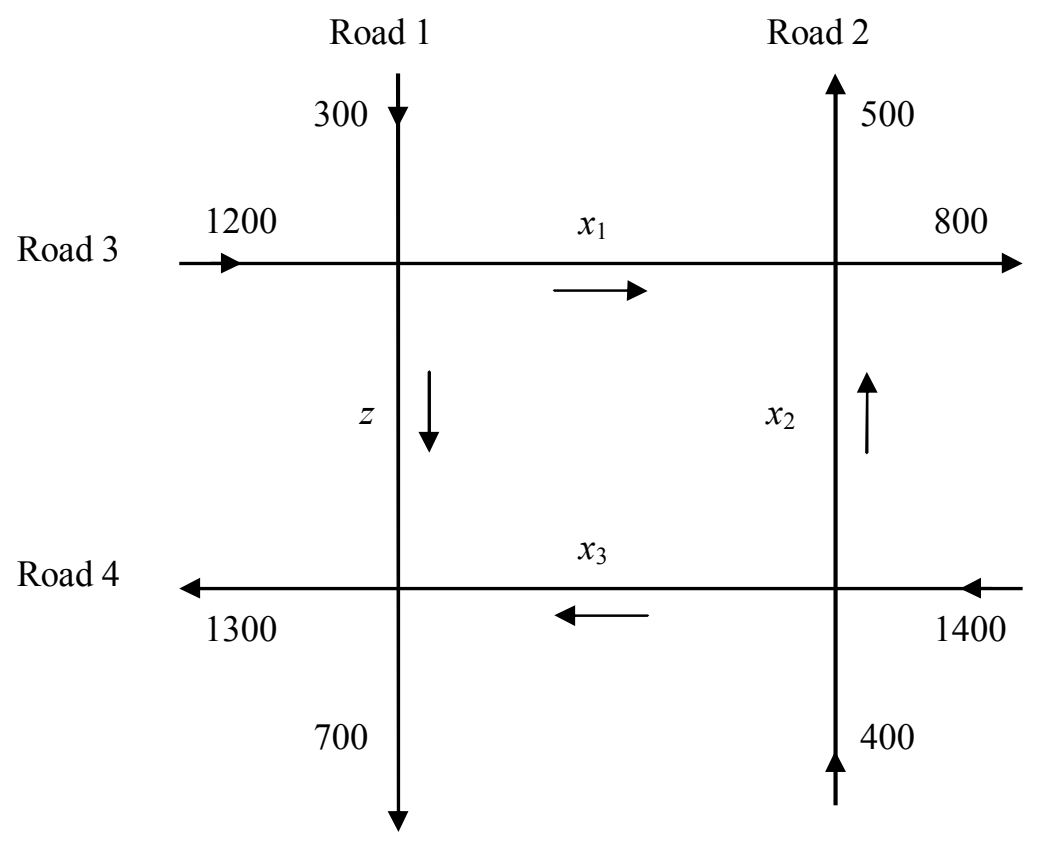

Figure 1. The downtown traffic flows of four roads.

To avoid traffic congestion, all traffic entering an intersection must also leave that intersection; i.e., the number of entering vehicles is equal to the number of leaving vehicles in each intersection in Figure 1. According to this condition to each of the four intersections in a clockwise direction beginning from the intersection between Road 1 and Road 3, the four linear equations based on the four intersections in Figure 1 can be obtained as follows:

$$
\begin{aligned}
& 1500=x_{1}+z \\
& 1300=x_{1}+x_{2} \\
& 1800=x_{2}+x_{3}
\end{aligned}
$$




$$
2000=x_{3}+z
$$

Thus, the system of the four linear equations in the three variables $\left(x_{1}, x_{2}, x_{3}\right)$ can be simplified into the following form:

$$
\begin{gathered}
x_{1}=1500-z \\
x_{1}+x_{2}=1300 \\
x_{2}+2 x_{3}=3800-z
\end{gathered}
$$

According to the $\mathrm{NN} z=400+I$, the system can also be represented by the following three neutrosophic linear equations:

$$
\begin{gathered}
x_{1}=1500-(400+I) \\
x_{1}+x_{2}=1300 \\
x_{2}+2 x_{3}=3800-(400+I)
\end{gathered}
$$

Then, there are the three neutrosophic linear equations:

$$
\begin{gathered}
x_{1}=1100-I \\
x_{1}+x_{2}=1300 \\
x_{2}+2 x_{3}=3400-I
\end{gathered}
$$

Thus, let us write the following neutrosophic matrices:

$$
A=\left[\begin{array}{lll}
1 & 0 & 0 \\
1 & 1 & 0 \\
0 & 1 & 2
\end{array}\right], X=\left[\begin{array}{l}
x_{1} \\
x_{2} \\
x_{3}
\end{array}\right], \text { and } B=\left[\begin{array}{c}
1100-I \\
1300 \\
3400-I
\end{array}\right]
$$

Hence, the system of neutrosophic linear equations can be written as the form of the neutrosophic matrix equation:

$$
A X=B .
$$

For the system of neutrosophic linear equations, we can therefore use the inverse matrix method to solve the neutrosophic matrix Equation (5).

Firstly, we obtain the inverse matrix of the neutrosophic matrix $A$ below:

$$
A^{-1}=\left[\begin{array}{ccc}
1 & 0 & 0 \\
-1 & 1 & 0 \\
0.5 & -0.5 & 0.5
\end{array}\right]
$$

Then, by multiplying both sides of Equation (5) by $A^{-1}$ (on the left), we can find the solution of the system:

$$
X=A^{-1} B=\left[\begin{array}{ccc}
1 & 0 & 0 \\
-1 & 1 & 0 \\
0.5 & -0.5 & 0.5
\end{array}\right]\left[\begin{array}{c}
1100-I \\
1300 \\
3400-I
\end{array}\right]=\left[\begin{array}{c}
1100-I \\
I+200 \\
1600-I
\end{array}\right]
$$

Clearly, the values of $X$ are NNs. In some real situation and/or requirement, when the indeterminacy $I \in[0,100]$ is considered as a possible range, the solution of the system is as follows:

$$
\left[\begin{array}{l}
x_{1} \\
x_{2} \\
x_{3}
\end{array}\right]=\left[\begin{array}{c}
{[1000,1100]} \\
{[200,300]} \\
{[1500,1600]}
\end{array}\right]
$$


Obviously, corresponding to the possible traffic flow $z=[400,500]$, we can obtain the ranges of the three traffic flows: $x_{1}=[1000,1100], x_{2}=[200,300]$, and $x_{3}=[1500,1600]$.

If the indeterminacy $I \in[200,400]$ is considered as a possible range, then the solution of the system is given as follows:

$$
\left[\begin{array}{l}
x_{1} \\
x_{2} \\
x_{3}
\end{array}\right]=\left[\begin{array}{c}
{[700,900]} \\
{[400,600]} \\
{[1200,1400]}
\end{array}\right] .
$$

Thus, corresponding to the possible traffic flow $z=[600,800]$, we can obtain the ranges of the three traffic flows: $x_{1}=[700,900], x_{2}=[400,600]$, and $x_{3}=[1200,1400]$. Hence, we usually obtain the possible ranges of the solution for $X$ in indeterminate problems, and then in actual applications we can choose some possible interval range [infI, supI] of the indeterminacy I corresponding to actual situations and/or requirements.

To show the effect of different values of indeterminacy, Table 1 demonstrates the traffic flows with respect to different ranges of indeterminacy $I \in[$ infI, supI]

Table 1. Traffic flows with respect to different ranges of indeterminacy $I$.

\begin{tabular}{ccccc}
\hline$I$ & $z$ & $x_{1}$ & $x_{2}$ & $x_{3}$ \\
\hline$I=0$ & 400 & 1100 & 200 & 1600 \\
$I \in[100,200]$ & {$[500,600]$} & {$[900,1000]$} & {$[300,400]$} & {$[1400,1500]$} \\
$I \in[200,300]$ & {$[600,700]$} & {$[800,900]$} & {$[400,500]$} & {$[1300,1400]$} \\
$I \in[300,400]$ & {$[700,800]$} & {$[700,800]$} & {$[500,600]$} & {$[1200,1300]$} \\
$I \in[400,500]$ & {$[800,900]$} & {$[600,700]$} & {$[600,700]$} & {$[1100,1200]$} \\
\hline
\end{tabular}

\section{Conclusions}

Based on indeterminate problems in the real world, this paper presented for the first time the concepts of neutrosophic linear equations and neutrosophic matrix, and introduced the neutrosophic matrix operations. Then, we proposed solving methods for neutrosophic linear equations, such as the substitution method, the addition method, and the inverse matrix method, for the system of neutrosophic linear equations (or neutrosophic matrix equation). Finally, an applied example about a traffic flow problem in a real scenario was provided to show the application and effectiveness of handling the indeterminate traffic flow problem by using the system of neutrosophic linear equations. A new effective way is provided for avoiding traffic congestion under indeterminate environments.

It is obvious that the neutrosophic linear equations proposed in this paper is not only the generalization of classical linear equations, but can also deal with real problems with determinate and/or indeterminate information. In the future, we shall extend these techniques to the modeling and analyses of engineering areas such as rock mechanics, engineering design, and engineering magament.

Acknowledgments: This paper was supported by the National Natural Science Foundation of China (Nos. 71471172, 61703280).

Conflicts of Interest: The author declares no conflict of interest.

\section{References}

1. Koukol, M.; Zajílková, L.; Marek, L.; Tulek, P. Fuzzy logic in traffic engineering: A review on signal control. Math. Probl. Eng. 2015, 2015. [CrossRef]

2. Zadeh, L.A. Fuzzy sets. Inf. Control 1965, 8, 338-353. [CrossRef]

3. Kaufmann, A.; Gupta, M.M. Introduction to Fuzzy Arithmetic; Van Nostrand Reinhold Company: New York, NY, USA, 1985.

4. Hanss, M. Applied Fuzzy Arithmetic: An Introduction with Engineering Applications; Springer: Berlin, Germany, 2005. 
5. Smarandache, F. Neutrosophy: Neutrosophic Probability, Set, and Logic; American Research Press: Rehoboth, DE, USA, 1998.

6. Smarandache, F. Introduction to Neutrosophic Measure, Neutrosophic Integral, and Neutrosophic Probability; Sitech: Craiova, Romania; Education Publishing: Columbus, OH, USA, 2013.

7. Smarandache, F. Introduction to Neutrosophic Statistics; Sitech: Craiova, Romania; Education Publishing: Columbus, OH, USA, 2014.

8. Ye, J. Multiple-attribute group decision-making method under a neutrosophic number environment. J. Intell. Syst. 2016, 25, 377-386. [CrossRef]

9. Ye, J. Bidirectional projection method for multiple attribute group decision making with neutrosophic numbers. Neural Comput. Appl. 2017, 28, 1021-1029. [CrossRef]

10. Kong, L.W.; Wu, Y.F.; Ye, J. Misfire fault diagnosis method of gasoline engines using the cosine similarity measure of neutrosophic numbers. Neutrosophic Sets Syst. 2015, 8, 43-46.

11. Ye, J. Fault diagnoses of steam turbine using the exponential similarity measure of neutrosophic numbers. J. Intell. Fuzzy Syst. 2016, 30, 1927-1934. [CrossRef]

12. Smarandache, F. Neutrosophic Precalculus and Neutrosophic Calculus; Europa-Nova: Brussels, Belgium, 2015.

13. Ye, J.; Yong, R.; Liang, Q.F.; Huang, M.; Du, S.G. Neutrosophic Functions of the Joint Roughness Coefficient (JRC) and the Shear Strength: A Case Study from the Pyroclastic Rock Mass in Shaoxing City, China. Math. Probl. Eng. 2016, 2016. [CrossRef]

14. Chen, J.Q.; Ye, J.; Du, S.G.; Yong, R. Expressions of rock joint roughness coefficient using neutrosophic interval statistical numbers. Symmetry 2017, 9, 123. [CrossRef]

15. Chen, J.Q.; Ye, J.; Du, S.G. Scale effect and anisotropy analyzed for neutrosophic numbers of rock joint roughness coefficient based on neutrosophic statistics. Symmetry 2017, 9, 208. [CrossRef]

16. Ye, J.; Chen, J.Q.; Yong, R.; Du, S.G. Expression and analysis of joint roughness coefficient using neutrosophic number functions. Information 2017, 8, 69. [CrossRef]

17. Ye, J. Neutrosophic number linear programming method and its application under neutrosophic number environments. Soft Comput. 2017. [CrossRef]

(C) 2017 by the author. Licensee MDPI, Basel, Switzerland. This article is an open access article distributed under the terms and conditions of the Creative Commons Attribution (CC BY) license (http:/ / creativecommons.org/licenses/by/4.0/). 